\title{
Los Efectos del High Performance Workplace Organization en la Productividad y en los Salarios de los Trabajadores
}

\author{
Adrián Todoli Signes*
}

Resumen:

Las nuevas prácticas de dirección de recursos humanos que se recogen bajo este genérico concepto de High Performance WorkplaceSystems (HPWO) son muy variadas, aunque todas ellas suelen incluir algún tipo de feedback, flexibilidad laboral, autocontrol por parte de los trabajadores, desaparición de los mandos intermedios, etc. En cualquier caso todas ellas tienen dos cosas en común que ha hecho que sean tan populares $y$ tan utilizadas en EEUU.

La primera es que su correcta aplicación en las empresas implica un aumento de la productividad de los trabajadores (ha quedado demostrado por diversos estudios Ichniowski 1996, Osterman 2006, Reich 2010). Y la segunda que requieren una alta cooperación entre el trabajador y la empresa (Freeman 2010).

Lo que no queda tan claro y es lo que está comunicación pretende abordar desde un plano teórico es si esta forma de "managment" implica un aumento de salario para los trabajadores.

Las primeras conclusiones dependerán de bajo qué hipótesis se actúa, puesto que en un mercado de trabajo completamente competitivo, todo aumento de la productividad debería repercutir en un aumento de los salarios. Si bien en un mercado de trabajo

\footnotetext{
* Investigador en Formación "Atracció al Talent". Depto. de Derecho del Trabajo y de la Seguridad Social. Universidad de Valencia. • adrian.todoli@uv.es
} 
completamente monopolístico, por el contrario, los aumentos de productividad irán a aumentar los beneficios del empresario.

Pero como la realidad no está hecha de extremos, en última instancia, dependerá del poder de negociación del trabajador o del sindicato, el que este pueda o no apoderarse de las ganancias de productividad en forma de aumento de salario. Cuestión que en este concreto supuesto de la HPWO es especialmente interesante puesto que como se ha dicho, su aplicación requiere un alto grado de cooperación entre el empresario y los trabajadores.

ABSTRACT:

The new practices of human resource management that are collected under this generic concept of High Performance Workplace Systems (HPWOs) are varied, although they all usually include some kind of feedback, labor flexibility, self-control by the workers, the disappearance of middle managers, etc. In any case all have two things in common that has made them so popular and as used in the US. The first is that its correct application in enterprises implies an increase in workerproductivity (Ichniowski 1996, Osterman 2006, Reich 2010). And the second that require high cooperation between the worker and the company.

What is less clear and is intended to address in this paper, from a theoretical level, is whether this form of management implies a wage increase for workers.

The first conclusions depend on that bypothesis acts as a fully competitive job market, any increase in productivity should result in any increase in wages. Although a labor market completely monopolistic, by contrast, increases in productivity will increase the profits of their employer.

However, the reality is not made of extremes, ultimately depends on the bargaining power of the worker or the union, this may or may not seize productivity gains in the form of salary increase. Issue in this particular case the HPWOs is especially interesting because as said, its application requires a high degree of cooperation between employers and workers.

PALABRAS CLAVE:

High Performance Workplace Systems, salario, sistemas salariales, productividad, resultados empresariales, estructura salarial, participación en beneficios, primas de productividad.

KEY WORDS:

High Performance Workplace Systems, wages, salary, wage systems, productivity, gainsharing, profit-sharing, wage structure. 


\section{INTRODUCCIÓN}

En la segunda mitad del S. XX las empresas comenzaron a incrementar la importancia dada a los recursos humanos, esto es una consecuencia lógica derivada de la globalización. El aumento de la competencia que sufren las empresas hace necesario ser eficiente en todas las facetas del proceso productivo, incluido en el manejo del capital humano. Así pues, la gestión estratégica de los recursos humanos ha sido definido como el conjunto de medidas y acciones desarrolladas con la intención de que la organización alcance sus objetivos (Wright y McMahan 1992; 298). De esta definición se desprende que la gestión de los recursos humanos es un medio para conseguir los fines de la empresa. Son un conjunto de medias que deben ir encaminadas a hacer más eficiente la gestión de los trabajadores para que estos sean más productivos, es decir mejorar la productividad laboral. Pero a su vez, el aumento de la productividad laboral es un medio para un fin, el cual debe ser mejorar la eficiencia de toda la compañía que ahora sí puede ser medida de diferentes maneras, a través del aumento de las ventas o de los beneficios, aumento de valor las acciones, etc...

Efectivamente, de acuerdo con Delery and Shaw (2001), hay al menos dos características que distinguen los nuevos modelos de gestión de los recursos humanos de los tradicionales. La primera es que la nueva gestión de recursos humanos debe tratar de mejorar la eficiencia de la empresa en general, es decir atendiendo a todos los aspectos de esta, y no solo a aspectos concretos como la forma de trabajar o el organigrama de la empresa. La segunda característica es el nivel de estudio. En el sistema tradicional se pretendía mejorar el puesto de trabajo y la eficiencia del trabajador desde una perspectiva individual. La nueva gestión asciende en el nivel de estudio y se centra en los equipos de trabajo o unidades de negocio. Como se ve, ambas características diferenciadoras en el fondo responden a una misma realidad y es el abandono de la particularidad en la gestión de los recursos humanos para alcanzar niveles más altos, con objeto de vincular más fácilmente la gestión de recursos humanos con las necesidades finales de la empresa. En este nuevo modelo, lo importante no es el trabajador individualizado y su rendimiento concreto, sino el rendimiento de los equipos de trabajo.De la misma manera los objetivos a perseguir no son solamente mejorar la eficiencia en el trabajo sino vincular las actividades de los trabajadores con los objetivos de la empresa.

De esta manera, los recientes estudios se han centrado en los llamados High Performance WorkplaceSistems (HPWO), las cuales son prácticas de gestión de los recursos humanos diseñadas para mejorar las habilidades de los trabajadores, su compromiso con la empresa, y la productividad de esta, como forma de que los trabajadores se conviertan en una ventaja competitiva sostenible para la empresa (Lawler, 1992, 1996; Levine, 1995; Pfeffer, 1998).

Este nuevo concepto engloba un conjunto de prácticas que todas tienen en común los objetivos arriba expuestos, y que responde a esta nueva forma de gestión de 
recursos humanos. Pero a la vez no existe ningún estudio, ni teórico (Lawler, 1992; Levine, 1995; Pfeffer, 1998), ni empírico (Arthur, 1994; Huselid, 1995) que recoja todas las formas que pueden adoptar estos HPWO. Así pues, la primera conclusión que se debe sacar sobre estas prácticas es que existe una gran variedad de ella que han sido interpretadas como "High performance". Becker y Gerhart (1996) detectaron que en el análisis de solo cinco estudios sobre los HPWO, se habían recogido un total de 27 prácticas distintas de las cuales solo cuatro de ellas eran comunes en más de tres estudios. Por tanto, los investigadores utilizan este HPWO como comodín para nombrar todas aquellas practicas que tiene por objetivo tener trabajadores más preparados (Skill-basedpay plan, jobrotation), mejorar el compromiso de estos con la empresa (profit-sharing, gain-sharing) o mejorar la productividad de esta (self-managedteam, employeeinvolvement).

Además de lo anterior, en este estudio se verá que en muchos casos es necesaria la adopción de dos o más practicas para que estas tengan un verdadero efecto, puesto que individualmente no producen cambios en la eficiencia de la organización. Esto es el llamado efecto sinergia, el cual parte de la idea de que con la puesta en práctica de dos o más practicas HPWO el resultado total es mejor que la suma de los dos individualmente, llegando al extremo de que una práctica individualmente no puede ser considerada High performance, pero si se conjunta con otras, el resultado final es suficientemente positivo para que entre en esta categoría (Levine y Tyson 1990; Weitzman and Kruse, 1990)

Esta falta de consenso sobre que prácticas que deben ser consideradas HPWO y cuáles no, imposibilita establecer una lista cerrada sobre ellas. A pesar de esto, en mi investigación he podido concretar una serie de prácticas, las cuales la mayoría de los investigadores considera que sí lo son.

\section{DIFERENTES PRÁCTICAS ENGLOBADAS EN EL CONCEPTO DE HIGH PER- FORMANCE WORKPLACE}

1) Total Quality Management (TQM): El Total Quality Management, es otro concepto indeterminado que nace más como filosofía de gestión que como técnica (Sullivan 1992). El TQM combina las enseñanzas de Deming (1986) y Juran (1989) sobre los procesos de control a través de la estadística y los valores de la industria japonesa sobre la importancia de la calidad del producto y le mejora continua. En Japón este movimiento comenzó a hacerse popular durante los años 50 durante la recuperación de la II guerra mundial. Mientras que en EEUU y Europa hubo que esperar hasta los años 80 para que el interés sobre estas técnicas comenzara a brotar, cosa que hizo precisamente por el éxito que las empresas Japonesas estaban teniendo en el mercado global (Kart, Kochan, Gobeille, 1983; Kart, Kochan, Weber, 1985). Las principales líneas de esta filosofía se basan en focalizar la gestión de recursos humanos hacia la satisfacción del consumidor a través del aumento de la calidad del producto. Se argumenta que un aumento de la calidad del producto disminuirá los costes de la 
empresa y permitirá a esta responder más rápidamente y con mayor eficiencia a la demanda de los consumidores.

Con esta línea de pensamiento, la TQM es considerada una cultura, en el sentido que intenta cambiar los valores de la empresa y los trabajadores, así como su comportamiento en múltiplesaéreas. Así pues, la responsabilidad de la dirección es asegurarse que se da prioridad a estos principios a lo largo de toda la empresa (Edward, Lawler, 1994; p 69).

De esta manera en la necesidad de que todos los trabajadores de la empresa estén imbuidos de estos valores respecto a la calidad del producto y centrados en las necesidades del consumidor es donde entra en juego la gestión estratégica de los recursos humanos. En el TQM se espera que los trabajadores pongan atención a los problemas de calidad del producto que salgan en su puesto de trabajo y reporten a la dirección tales problemas, e incluso se espera de ellos el aporte de una posible solución. No solo esto sino se espera que los trabajadores acepten y colaboren en la continua mejora de la cultura y que aporten formas de mejorar la calidad del producto. Bajo esta filosofía de trabajo recae sobre el empleado la responsabilidad de mejorar la organización del trabajo, la resolución de conflictos, la calidad del producto y la satisfacción del cliente.En definitiva es el trabajador el que tiene la llave para la mejora de la eficiencia empresarial.

Conocida la filosifia, las técnicas para la consecución de estos objetivos arriba expuestos parten del llamado "Qualitycircles" (Ishkawa, 1980, 1985) basado en utilizar a los equipos de trabajo como fuente de información de los problemas, incentivando que los propios trabajadores hagan sugerencias y resuelvan los problemas en el propio equipo. Pero como hemos visto, en la filosofía de las TQM las aportaciones de los trabajadores no deben reducirse a cuestiones del puesto de trabajo sino que las sugerencias deben alcanzar a los métodos de trabajo, a los procesos de producción e incluso a los sistemas de dirección de la empresa. El énfasis se debe poner sobre todo en la simplificación del trabajo y su codificación en actividades sencillas y cuidadosamente especificadas (Edward, Lawler, 1994; p 70).

En definitiva esta técnica que comprende multitud de diferentes actuaciones, se basa en recoger la máxima información posible de los trabajadores con objeto de mejorar la calidad del producto y la satisfacción del cliente ${ }^{1}$.

2) Self-Management: La autonomía en los equipos de trabajo, se basa en dar a los miembros de este la autoridad sobre decisiones que tradicionalmente dependían de los supervisores, como por ejemplo cómo realizar las tareas, el orden de realización

1. Este es un ejemplo claro del nuevo tipo de gestión de recursos humanos, que se ha planteado en el primer epígrafe, donde se abandona la tradicional gestión individualizada y que pretende mejorar solo el flujo de trabajo a favor de una gestión en equipos de trabajo que pretende mejorar la eficiencia de toda la empresa, en este caso medida en términos de satisfacción del cliente. 
de cada una de las tareas, etc., incluso llegando en niveles más avanzados a tener potestad para elegir que tareas realizar. Esto encaja perfectamente en las nuevas teorías respecto a que la excesiva jerarquización reduce la creatividad del trabajador. Por ello, muchas empresas están tomando conciencia de esto haciendo desaparecer a los mandos intermedios, horizontalizando la empresa, y permitiendo que los trabajadores actúen más libremente (Tropman,2001; p 154). Se espera que con este aumento de la autonomía los empleados sean capaces de mejorar sus niveles de productividad. En cualquier caso, con esto la empresa no abandona cualquier control sobre los empleados sino que deja en manos del los compañeros de equipo el control de los individuos concretos.

3) Meetings: Esta técnica de HPWO se basa en programar reuniones de manera regular donde discutir los problemas de trabajo. Estos "meetings" pueden ser supervisados con un mando intermedio, o bien dejar que los equipos de trabajo discutan libremente los problemas e incluso busquen y apliquen por ellos mismos las soluciones aportadas.

4) Job-Rotation: La rotación en los puestos de trabajo pretende la flexibilidad laboral, haciendo que los trabajadores aprendan a manejarse en diferentes posiciones de la empresa con dos objetivos principales. El primero es efectivamente aumentar la flexibilidad del factor trabajo permitiendo que el empresario pueda disponer de trabajadores capaces de asumir todas o la gran mayoría de funciones en la empresa, y de esta manera pueda gestionar de manera más eficiente a los trabajadores. El segundo objetivo de la rotación de los trabajadores en los puestos de trabajo, es permitir que estos conozcan todos los pasos del proceso productivo permitiendo que tengan una visión global de la empresa, facilitando de esta manera que realicen sugerencias sobre cómo mejorar la productividad y la eficiencia de toda la empresa.

5) Skills-basedpay plan: La remuneración por competencia es un sistema salarial basado en recompensar a través de aumentos salariales a los trabajadores por las habilidades, conocimientos, o competencias que estos poseen o adquieren en un momento dado. Así pues, a diferente número o nivel de habilidades corresponderá un diferente nivel salarial. En este sistema la demostración de tener la competencia objeto de remuneración es suficiente para recibir el complemento salarial correspondiente, sin que sea necesario realizar un trabajo que requiera utilizar esa competencia (Henderson, 2006; p 300). Es decir, se remunera el mero hecho de tenerla o adquirirla, y no el hecho de tener que utilizarla. En objetivo de este sistema salarial es tener a trabajadores en continua formación y preparados. Este sistema responde a la filosofía de que son los trabajadores los que deben aportar innovación a la empresa, por lo que invertir en la formación de estos es invertir en la competitividad de la empresa. Ahora bien, este es uno de los casos más claros en los que será necesaria la combinación con otras técnicas para aportar resultados. Un trabajador ultracuali- 
ficado si no se le permite aportar sus sugerencias a través de sistemas como TQM o el self-management probablemente no resulte productivo para la empresa. Es más, probablemente la empresa esté gastando mucho dinero en la formación de un trabajador sin sacarle rendimiento lo que acabará aumentando los costes laborales sin recibir contraprestación alguna en forma de beneficios (Tropman,200;p 157). E igual funciona en sentido contrario, las técnicas de TQM o self-management pueden aportar muy poco a la eficiencia de la empresa si estos trabajadores haciendo sugerencias no están debidamente preparados y formados para ello.

6) Profit-sharing: La participación en beneficios de los trabajadores se basa en compartir con estos las ganancias derivadas de las mejoras en el rendimiento de la empresa. Se basan en hacer depender el salario del trabajador con la eficiencia empresarial o con los objetivos de la empresa. De esta manera, el salario del trabajador puede ir indexado a los ingresos de la empresa, a la productividad, a las ventas, a los beneficios o incluso al valor de las acciones de esta. El objetivo de esta técnica es que el trabajador comparta los mismos intereses que la dirección de la empresa, haciendo desaparecer, al menos en parte, el conflicto social. Otra ventaja de este sistema es que el trabajador tendrá grandes incentivos en el buen funcionamiento de la empresa, haciendo que otras técnicas como los meetings o el TQMmejoren la productividad. Es decir, con esta técnica hace que los trabajadores sean los primeros interesado en que las cosas mejoren en la empresa, por tanto, tomando conciencia de esta necesidad realizarán mejores aportaciones en los meetings etc. Está demostrado que el Profit-sharing por sí mismo sin permitir algún nivel de participación de los trabajadores en la gestión es ineficiente (Cappell, and Neumark,2001; p 749). Cuestión sobre la que recae todo el peso de la lógica, puesto que por muy interesados que estén los trabajadores en mejorar el rendimiento de la empresa, si esta no se lo permite mediante otras técnicas de participación de los trabajadores en la gestión de la empresa, no habrá mejora alguna.

Respecto a los estudios económicos que relacionan estas prácticas con un aumento de la productividad del trabajo todos dan resultados positivos. Los estudios de Arthur (1994);MacDuffie (1995); Koch and McGrath (1996), Delery y Doty, (1996) Ichiowski Shaw, y Premushi, (1997); Delery, Gupta, y Shaw (1997), Cappelli, y Rogovsky, (1998),Ichniowski y Shawn (1998)Appelbaum, Eileen, Thomas Bailey, Peter Berg, and ArneKalleberg. (2000), Guthie (2001) Datta, Guthrie, y Wright (2005) demuestran que existe una alta correlación entre las HPWO y la productividad de factor trabajo.

Por otra parte, también hay estudios que buscan relacionar estas técnicas con el rendimiento empresarial, ya que como establece Cappell, and Neumark, (2001, p 739) es posible que la productividad del trabajo aumente sin que necesariamente aumenten los beneficios empresariales. Los beneficios obtenidos por el aumento de la productividad puede que no vayan al bolsillo del empresario sino que repercuta 
en una disminución del precio del producto, o bien en que haya un mayor reparto de la rentas del capital entre los trabajadores y el empresario, obteniendo los primeros una mayor parte del pastel, que haga que los beneficios finales del empresario no aumenten.

A este respecto,Easton and Jerrell (1998) estudiaron la introducción de los programas TQM a través de comparar los resultados de empresa que habían adoptado este tipo de técnica y otras que no, dejando todas las demás variables constantes. En este estudio los autores notaron que las empresas que adoptaron estas técnicas obtenían mejores resultados en forma de aumento del valor de las acciones (financial performance) que las que no. En el mismo sentido CumbsLiu, Hall, y Ketchen (2006) encuentran relación positiva entre esta técnica y el rendimiento empresarial.

Otro estudio analizando la participación del empleado (meetings, TQM, selfmanagement) y planes de compensación por productividad (profit-sharings y gainsharing) encontró que estas técnicas aumentaban los salarios por debajo del valor que añadían en el rendimiento empresarial. Llegando a la conclusión de en el resultado final la empresa mejoraba con la aplicación de estas técnicas (Cook, 1994).

Por otra parte, Cappell, and Neumark, (2001)p 766 admitiendo que estas nuevas técnicas de gestión de los recursos humanos aumentan la productividad del factor trabajo llega a la conclusión de que esto no tiene porque mejorar el rendimiento empresarial. En su estudio detecta un aumento de los costes laborales que en algunos casos superan las ganancias en productividad. Por ejemplo para el caso de las TQM y profit-sharing establece que el aumento del coste del trabajo es del 10.4\% mientras que la productividad solo aumenta 5.2\%.Por otra parte establece que el profit-sharing en conjunto con los meetings, provocan una aumento de los costes laborales del 7,4\% y un $12.2 \%$ de aumento de la productividad. En base a estos datos el estudio concluye que el aumento de los costes laborales, entendidos como aumentos salariales, hacen el rendimiento de la empresa no mejore.

A mi entender, este estudio pretende poner en duda la utilidad de estas técnicas de gestión de recursos humanos, afirmando que no siempre son beneficiosas para el empresario. El problema de este planteamiento es que se olvida que el aumento de la productividad es lo que hace avanzar la economía. El criterio a valorar sobre la bondad o no de esta técnica no debería ser los beneficios finales sino la productividad. Con esa mentalidad los avances de tecnología (los que más aumentan la productividad) no hubieran existido. La teoría de la competencia perfecta establece que todos los aumentos de productividad van directamente a reducciones del precio del producto sin aumento de beneficios para el empresario, así pues en base a esto no tendría sentido inversión alguna. Los empresarios deberían colusionar y no invertir.

Pero en la práctica el empresario que no invierte en tecnología, y no recibe los aumentos de productividad de esta, se convierte en la empresa más cara del mercado, perdiendo con ello sus clientes. Es decir, pierde su ventaja competitiva. De la misma 
manera, ocurre con estas técnicas, aquel empresario que no las adopte, una vez demostrado que aumentan la productividad, se arriesga a perder la ventaja competitiva que estos trabajadores pueden darle. Y no solo eso sino que se arriesga a perder a los propios trabajadores que tendrán incentivos para ir a trabajar a otras empresas con mejores salarios que los aumentos de la productividad les ha permitido pagar.

Así pues, se debe admitir que si estas técnicas aumentan los costes laborales, no lo hacen como algo patológico del sistema, sino como parte del sistema en el cual, con la democratización de los sistemas de relaciones laborales, para mejorar y crear una ventaja competitiva en la empresa es necesario que los intereses de los trabajadores y del empresario vayan juntos, siendo la mejor manera para conseguirlo, la repartición de las ganancias de la productividad. Será necesario, por tanto, que el trabajador reciba parte de los beneficios generados por un aumento de la productividad si el empresario quiere que este se produzca.

Pero antes de avanzarnos en conclusiones analicemos ahora los efectos que provocan estas técnicas en los salarios de los trabajadores.

\section{HPWO Y SALARIOS}

Una vez establecido que las técnicas de HPWO aumentan la productividad de la empresa, ahora se pasa a estudiar cómo se reparte esa generación de riqueza en el seno esta. Las posibilidades son dos, a) la primera sería que ese aumento de la productividad se produjera en todas las empresas del sector, por lo que para mantener la cuota de mercado frente a sus competidores la empresa se viera obligada a reducir los precios, al igual que el resto de empresas por lo que el excedente creado por la productividad iría a manos de los consumidores en forma de un producto de menor precio. b) La segunda se basa en la creación de una ventaja competitiva en la empresa, que la diferencia de las demás, en tal caso la empresa podría bajar el precio del producto (mayor productividad menor coste medio del producto) consiguiendo con ello un aumento en las ventas y, con ello, una aumento de los ingresos (Freeman, 2010; p 62). Ahora bien, en esta segunda opción, el aumento de los ingresos puede tener diferentes fines. Por una parte, la empresa puede dedicar esos ingresos a aumentar la inversión de la empresa. También puede dedicarlos a pagar las deudas, a crear puestos de trabajo, repartirlo entre las accionistas en forma de dividendos, aumentar la retribución del equipo directivo, aumentar los salarios de los trabajadores que provocaron ese aumento de la productividad en un primer momento, o bien aumentar los salarios de todos los trabajadores de la empresa (los involucrados en las HPWO y los que no). Es decir, en esta segunda opción (b), que es la más probable, existe un aumento de la riqueza en la empresa derivado de la mejora en la productividad, pero en que se transformará ese excedente depende de múltiples factores. 
Así pues, lo expuesto hasta ahora sirve para cualquier tipo de aumento de productividad en la empresa, es decir, la aparición de nueva tecnología que aumente la productividad se repartirá conforme se ha establecido. Pero las particularidades de las técnicas de la HPWO hacen que existan ciertas especialidades que deberían hacer decantar la balanza hacia un aumento de los salarios al menos a nivel teórico. Las razones son las siguientes.

1) Para aplicar las técnicas de HPWO se requieren trabajadores mejor preparados. Para que un grupo de trabajadores pueda tener autonomía se requiere que tenganuna formación mayor que la que necesita un trabajador bajo estricta supervisión de la dirección. De la misma manera para realizar la rotación en los puestos de trabajo es necesaria una formación mayor que para realizar un único trabajo. Incluso en el caso de los meetings y los TQM cuanta mayor formación tenga en trabajador mejores serán las suegerencias realizadas por este. Así pues, la alta formación de estos trabajadores les hará muy atractivos para las empresa de la competencia, por lo que si el empresario desea mantenerlos deberá retribuirlos con salarios de eficiencia, por encima de los salarios de mercado para su tipo de formación.

2) Está demostrado que las empresas que adoptan las técnicas de HPWO son más propensas a aumentar su inversión en formación del trabajador por las mismas necesidades antes expuestas (Osterman, 1995; Lynch y Black 1998). Así pues, el gasto realizado en la formación de estos trabajadores es una razón más para querer mantenerlos en la empresa y, por ello, pagar salarios más altos.

3) El aumento de la productividad provocado por estas técnicas viene del feedback proporcionado por los propios trabajadores, esto puede hacer que los trabajadores se sientan merecedores de una recompensa por ello. Es decir, los trabajadores pueden sentirse injustamente tratados si su esfuerzo para mejorar la productividad de la empresa no se corresponde con aumentos salariales. En caso de que sea una nueva tecnología la que aumente la productividad de la empresa esto no ocurre, los trabajadores pueden sentir que es el empresario el que ha hecho la inversión en nueva tecnología por lo que es justo que el excedente provocado por ese aumento de productividad vaya a los bolsillos del empresario en forma de beneficios, pero en el caso de las HPWO es el trabajador el que proporciona ese aumento de la productividad y probablemente se sienta merecedor de una recompensa en forma de mejora salarial. Ahora bien este sentimiento se convertirá en una realidad o no dependiendo del poder de negociación que tengan los trabajadores respecto a su empresario. De ahí los resultados obtenidos por Black, Lynch y Krivelyova (2004), los cuales detectaron que solo en empresas sindicalizadas la implantación de técnicas HPWO implicaban un aumento de salarios para el trabajador.

4) El cuarto motivo que puede provocar que el aumento de la productividad se convierta en aumento de salarios es el incremento de poder de negociación individual de los trabajadores. con independencia de si con anterioridad los trabajadores tenían 
o no poder de negociación dentro de la empresa (a través de la sindicalización o de representantes de los trabajadores), tras la implantación de estas técnicas este poder habrá aumentado. Todos los sistemas de HPWO requieren, de una u otra manera, la colaboración de los trabajadores. Es más, el existo de estas técnicas depende de los feedbacks dados por el trabajador, así como las sugerencias que este da para mejorar el proceso productivo (TQM, meetings). De esta manera, el trabajador es consciente de que la empresa depende más de su involucración en el proceso productivo y de que además esta saca un mayor provecho de su trabajo, por lo que el trabajador puede asegurarse un mayor porcentaje de las ganancias en productividad en forma de aumento de salarios. Del mismomodo, la empresa sabe que si quiere la colaboración del trabajador para aumentar la productividad deberá recompensarle por ello con una repartición de las ganacias (profit-sharing, gain-sharing).

A pesar de estas hipótesis arriba planteadas los estudios realizados respecto a la transformación del aumento de la productividad en mejoras salariales no son concluyentes. Appelbaum, Bailey, Breg, and Kalleberg (2000) en el estudio de tres industrias diferentes (industria del metal, de textil y de instrumentos médicos) encontraron que las técnicas de HPWO aumentaban los salarios en las dos primeras pero no en la tercera. Hunter y Lafkas (2003) estudiando el sector bancario la parte de atención al consumidor, encontraron que los QualityCircles (parte de las TQM), provocaba aumentos salariales. Como se ha visto ya Cappelli y Neumark (2001) estudiando las empresas manufactureras encuentra relación entre estas técnicas y el aumento de los costes salariales. También Osterman (2006) estudiando la industria manufacturera encontró que la implantación de técnicas de HPWO aumentaba los salarios de todos los trabajadores y también las retribuciones del equipo directivo. Por su parte, el mismo autor años antes (Osterman, 2000), estudiando empresas americanas de todos los sectores, de al menos 50 trabajadores, encontró que los salarios no aumentaban por la introducción de los sistemas de HPWO. De la misma manera,Handel y Gittleman (2004), estudiando los mismos datos que el estudio de Osterman, (2000), tampoco entroncaron relación entre el uso de estas nuevas técnicas de gestión de recursos humanos y los salarios. Incluso se ha llegado a encontrar evidecias de que las técnicas de HPWO pueden realmente reducir el salario de los trabajadores (Rosen 1993). Por último, un interesante estudio de Black, Lynch y Krivelyova (2004) encontraron que los sistemas de HPWO solo aumentaban los salarios en las empresas sindicalizadas.

Así pues, de los estudiosempíricos no se pueden sacar conclusiones certeras. A diferencia de la relación entre los sistemas de HPWO y la productividad donde sí parece haber una relación directa y empíricamente demostrable, en el caso de la relación entre la HPWO y lo salarios esta relación no es tan clara.

A mi entender, las razones por las que el aumento de la productividad provocado por la implantación de las HPWO no siempre se transforman en aumentos salariales 
es porque esta relación depende de la distribución del poder en la empresa (Osterman, 2006; p 190). El aumento de la productividad lo único que hace es que aumente la capacidad de la empresa de pagar más a sus trabajadores, pero esa capacidad solo se transformará en un efectivo aumento salarial si el poder de negociación de los trabajadores, vía sindicalización o vía necesidades de colaboración del trabajador aumentan. Pero no son estas las únicas variables. También está demostrado empíricamente que estas técnicas aumentan los despidos en la empresa (Freeman 2010, p 63). La desaparición de la necesidad de los mandos intermedios provoca que la empresa tenga excedente de mano de obra, así pues el miedo provocado por los despidos puede reducir el poder de negociación de los trabajadores, haciendo que sea el empresario el que se quede con el excedente (Rosen, 1993). También es posible que el aumento del poder de negociación que los trabajadores obtienen con la implantación de estas técnicas prefiera ser utilizado para otras cosas y no para los aumentos salariales. Quizá los trabajadores prefieren reducir la jornada, manteniendo el mismo salario, o prefieren que la empresa se comprometa a mantener los puestos de trabajo, intercambiando seguridad por salario (Wenchuan, Guthrie, Flood,MacCurtain, 2009; p 110).

Es decir, lo único que se puede mantener con seguridad es que las técnicas aumentan la productividad, y que la transformación de esa productividad en aumentos salariales dependerá de la distribución del poder en la empresa, pudiendo afirmar también que este tipo de técnicas son propensas a aumentar el poder individual de los trabajadores derivado de las necesidades de colaboración por parte de los trabajadores que es necesaria para la efectividad de las propias técnicas. De aquí la necesidad de que estas técnicas cuenten con la aprobación de los propios trabajadores. Black y Lynch(2001, p. 439), establecen que la introducción de las técnicas HPWO por si solas no son suficientes, sino que necesario para obtener significativos aumentos de productividad que se combinen con la participación de los trabajadores en la toma de decisiones, lo que irremediablemente requiere un aumento de poder de negociación de los trabajadores en la empresa. También se ha probado que para que estos sistemas sean efectivos es necesario que el trabajador sienta que es recompensado por su mayor esfuerzo, y que parte de las ganancias obtenidas gracias a estas técnicas repercuta en su bienestar (Black y Lynch 2001; p 444). Lo cual puede hacerse, como normalmente se hace, por sistemas de participación en beneficios, o bien con reducciones de jornada o mejora de la seguridad en el empleo etc.

\section{LA SINDICALIZACIÓN DE LA EMPRESA Y LOS SISTEMAS DE HPWO}

Debido a la importancia detectada en este estudio que tiene el poder de negociación del trabajador a la hora de que este obtenga parte de las rentas creadas en la instauración de los sistemas de HPWO, considero importante analizar qué efectos tiene implantación de un sindicato fuerte o de una arraigada representación de los trabajadores en la empresa. 
La teoría del sindicalismo se basa en que la unión de los trabajadores aumenta el poder de negociación de estos frentes al empresario. En esto se basa la negociación colectiva, llegando en sistemas legales como el español donde se da preferencia a la negociación colectiva para establecer ciertas condiciones del contrato de trabajo como por ejemplo la estructura salarial (art. 26.3 ET). Así pues, esta teoría es coherente con los resultados obtenidos por Black, Lynch y Krivelyova (2004) los cuales encontraron que los sistemas de HPWO solo aumentaban los salarios en las empresas sindicalizadas. Es decir, solo a través del poder de negociación de los trabajadores pueden estos apoderarse de parte de los rendimientos que las técnicas HPWO proveen. Pero este no es el único efecto que provoca la sindicalización de la empresa con respecto a estas técnicas.

Como establece Malcomson (1983) las implantación de estas técnicas puede ser mejor entendida por parte de especialistas del sindicato que por los trabajadores individuales, resultando más fácil que una empresa sindicalizada sea más propensa a aceptar este tipo de cambios. También, como se ha dicho, la implantación de estas técnicas puede provocar despidos en la empresa, por tanto, los trabajadores en teoría estarán en contra de estas nuevas medidas salvo que el sindicato esté en posición de garantizarles la seguridad en el empleo (Black y Lynch, 2001; p 436).Levine y Tyson (1990, p 210)establecen que los trabajadores serán reacios a cooperar con técnicas que incrementen la eficiencia si tienen miedo de que haciéndolo ponen en peligro su puesto de trabajo.

Por otra parte, se ha argumentado que estas técnicas aumentan el poder de negociación individual del trabajador haciendo que el sindicado deje de ser útil. En base a esto hay autores que defienden que el sindicato tiene incentivos para "sabotear" este tipo de técnicas (Guest, 1995). La teoría detrás de esta afirmación se basa en que con la participación en beneficios (profit-sharing) y la participación en la gestión (self-managementteams, TQM, meetings) la voz de los trabajadores pasa a ser directamente escuchada y valorada por el empresario, haciendo innecesario al sindicato. Asi pues, en base a esto se prevé que el sindicato pierda afiliación y, por tanto, vaya en contra de los intereses del sindicato cooperar en este tipo de medidas (Fisher 1997; Godard 2004; Kizlos y Reshef, 1997). Además, se argumenta que estas técnicas de participación de los trabajadores en la gestión hacen que estos asuman responsabilidades que anteriormente lo hacían los supervisores y la dirección de la empresa erosionando con ello la solidaridad de clase (Kizilos y Reshef, 1997). Es decir, la función clásica del sindicato ha sido históricamente reducir la discrecionalidad del empresario, a través del establecimiento de reglas detalladas o normas legalmente vinculantes (negociación colectiva). Así pues, si el sistema HPWO se basa en aumentar la autonomía del trabajador, reduciendo la jerarquización y aumentando el poder de decisión del trabajador individual, el sindicato se queda sin su papel tradicional (Wenchuan, Guthrie, Flood,MacCurtain, 2009; p 112). Fiorito (2001) sugiere que estas 
políticas de recursos humanos suelen reducir la insatisfacción del trabajador, que es la primera causa de sindicalización. Incluso hay estudios que sugieren que algunas empresas adoptan estas técnicas en parte como forma de reducir el poder del sindicato en la empresa (Godard, 1998)².

Pero a mi entender, los intereses que hay detrás de estas técnicas de recursos humanos son consistentes con los intereses y las demandas del sindicato ${ }^{3}$. El objetivo de este siempre debe ser mejorar la posición del trabajador en la empresa, mejorando sus condiciones de trabajo. Así pues, la adopción de este tipo de técnicas puede considerarse una victoria del sindicato sobre la empresa ${ }^{4}$. Es más, dado que los estudios demuestran que la cooperación del sindicato es importante para mejorar los resultados de este tipo de técnicas (Cook, 2004), la dirección empresarial deberá negociar con el sindicato o los representantes de los trabajadores la implantación de ellas. De este modo, a los ojos de los trabajadores estas nuevas técnicas vendrán del acuerdo entre el sindicato y la empresa (negociación colectiva), percibiendo con buenos ojos la actuación del sindicato gracias a los beneficios que estas nuevas técnicas provén para el bienestar del trabajador y la productividad de la empresa. Si a esto se le suma que para que esos aumentos en productividad se transformen en salarios hará falta la intervención de nuevo del poder negociador del sindicato, una vez más, la figura de este se convertirá en una activo importante para el trabajador.

A mi juicio, estas técnicas deben ser aprovechadas por los sindicatos para alejarse de las tradicionales confrontaciones entre el capital y el trabajo, para así pasar a una nueva forma de actuar en la que se favorezca la cooperación entreambos 5 .

En fin, a mi entender aquel sindicato que por miedo a perder su influencia sobre los trabajadores se resista a la implantación de este tipo de técnicas que mejorar la posición del trabajador en la empresa, no estaría haciendo bien su papel, y como en el libro de Mary Shelley, el sindicato se habría convertido en una maquina que lucha contra los intereses de su creador.

\section{CONCLUSIONES}

Las conclusiones obtenidas en este estudio se pueden resumir de la siguiente manera.

2. Aunque este estudio también demuestra que este es un objetivo menor en comparación a los verdaderos objetivos de la empresa como la reducción de costes y el aumento de la calidad del producto fabricado por la empresa.

3. En el mismo sentido, Kochan 1995; Kochan y Osterman 1994; Rubenstein y Kochan 2001; Machin y Wood 2005.

4. Aunque en el fondo sea un "win-win" y tanto la empresa como los trabajadores salgan ganando.

5. En el mismo sentido Roche and Geary(2002). 
La primera es que existe gran diversidad de técnicas que se engloban bajo la idea de las High Performance, pero todas ellas tiene en común la búsqueda de mejorar las un habilidades de los trabajadores, su compromiso con la empresa, y la productividad de esta.

La segunda conclusión es que tanto teórica como empíricamente se demuestra que existe una relación entra las llamadas técnicas High Performance y la productividad del trabajador. Siendo necesario en algunos casos la combinación de dos o más técnicas para apreciar esos aumentos de productividad o para mejorarlos (sinergias). Los ejemplos más destacados son la necesidad de combinar los meetings y los TQM donde se espera feedback por parte del trabajador con profit-sharing con objeto de que el trabajador tenga incentivos para colaborar en estos feedbacks. Otro ejemplo es la necesidad de combinar trabajadores formados (skill-basedpay plan) con los selfmanagementteams, puesto que si los trabajadores no están suficientemente preparaos, la desaparición de los mandos intermedios puede provocar más perjuicios que ventajas.

Tercero respecto a la relación entre estás técnicas de gestión de recursos humanos y los salarios. La teoría nos dice que deberían estar positivamente correlacionados, estas técnicas implican una mayor participación de los trabajadores en la empresa y también requieren de ellos una colaboración cuestiones que deberían ser recompensadas en forma de aumentos de salarios. Pero los estudios empíricos no siempre respaldan esta opción.

Los factores que provocan que los beneficios obtenidos de por la implantación de estas técnicas no vaya acompañado de un aumento en los salarios se explican por varias razones. La primera es porque los trabajadores pueden estar interesados en ser recompensados de otras maneras que no sean con aumentos salariales, como por ejemplo obteniendo mayor seguridad en el empleo.

El segunda factor es que es posible la inexistencia de suficiente poder negociador de los trabajadores que consigue extraer pasa sí parte de ese excedente generado con el aumento de la productividad. Lo que ocurre en este caso es que los trabajadores tendrán poca motivación para colaborar con el empresario en estas técnicas puesto que ellos no van a obtener beneficio alguno de ellas, pudiendo incluso "sabotearlas" con un mecanismo similar al frenado obrero que estudiaba Taylor.

Así pues, debido a que para obtener el máximo resultado de estas técnicas queda empíricamente probado que es necesaria la colaboración del trabajador, es absolutamente necesario que este reciba parte de los beneficios de su implantación, tenga o no el trabajador poder de negociación para obtenerlo. La negación de esa parte del excedente solo producirá la falta de colaboración de este y, por tanto, que se reduzca con ello el excedente. Asi pues, con el "profit-sharing" el empresario no está "reglando" unas rentas que podría quedarse en su propio beneficio, sino que sin la implantación de un sistema de reparto de los beneficios obtenidos por el aumento 
de la productividad que estas técnicas provocan, el propio aumento de productividad no se produciría.

En fin, de este estudio se obtiene la conclusión de que tenga o no el trabajador poder negociador para sustraerle al empresario parte de la productividad obtenida, es inherente a este tipo de técnicas para que funciones que haya una compensación para el trabajador.

\section{BIBLIOGRAFÍA}

Appelbaum, Eileen, Bailey Thomas, Berg Peter, y Kalleberg Arne, (2000): Manufacturing Advantage: Why High-Performance Work Systems Pay Off, Ithaca, N.Y. Cornell University Press.

Arthur, Jeffrey B. (1994): "Effects of Human Resource Systems on Manufacturing Performance and Turn over." Academy of Management Journal, V. 37, $\mathrm{N}^{\circ} 3$, pp. 670-688.

Batt, Rosemary, (1999): "Work Organization, Technology, and Performance in Customer Service and Sales.",Industrial and Labor Relations Review, Vol. 52, № 4 , pp. 539-561.

Becker, Brian, y Grehart Barry, (1996): "The impact of Human Resource Management on Organizational Performance: Progress and Prospects" Academy of Management Journal, V. 39, n4, pp 779-801.

Black, Sandra, Lynch Lisa, y Krivelyova Anya, (2004):»How Workers Fare When Employers Innovate." Industrial Relations, V. 43, No 1, pp. 44-66.

Cappelli Peter, and Neumark David., (2001): "Do "High-Performance" Work Practives Improve Establishment-Level Outcomes?”,Industrial and Labor Relations Review, v. 54, n'4, pp737-775.

Cappelli, Peter, y Nikolai Rogovsky, (1998): "Employee Involvement and Organizational Citizenship: Implications for Labor Law Reform and Lean Production.", Industrial and Labor Relations Review, V. 51, No 4, pp. 633-653.

Combs, James, Liu Yongmei, Hall Angela, y Ketchen David., (2006): "How Much Do High-performance Work Practices Matter? A Meta-Analysis of Their Effects on Organizational Performance.”,Personnel Psychology, V. 59, nº 3, pp. 501-528.

Cooke, William N. (1994): "Employee Participation Programs, Group-Based Incentives, and Company Performance: A Union-Nonunion Comparison." Industrial and Labor Relations Review, V. 47, n 4, pp. 594-609. 
Datta, Deepak K., Guthrie James P., y Wright Patrick M., (2005): "HRM and Labor Productivity: Does Industry Matter?”, Academy of Management Journal, V. 48, no 1 pp. 135-145.

Delery, J. E., \& Shaw, J. D., (2001): "The strategic management of people in work organizations: Review, synthesis and extension”, en Rowland K.M. y Ferris G.R. (Eds.), Research in personnel and human resource management, Greenwich, Emerald Group Publishing Limited.

Delery, John E., Gupta Nina, y Shaw Jason D., (1997): "Human Resource Management and Firm Performance: A Systems Perspective.”, Mimeograph, University of Arkansas.

Delery, John E., y Doty D. Harold., (1996): «Modes of Theorizing in Strategic Human Resource Management: Tests of Universalistic, Contingency, and Configurational Performance Predictions.", Academy of Management Journal, V. 39, n. 4, pp. 802-835.

Deming, W.E, (1986): Out of crisis, Cambrige, MIT press.

Edward E, Lawler III, (1994): "Total quality Management and Employee Involvement: Are they compatible?", The Academy of Management Executive, V. 8 n¹, pp 68-76.

Feeman Donald E., (2010): "Wage Effects of High Performance Workplace Practices", in Feeman Donald E., Essays in Labor Economics, PHD thesis Unpublished. P. 60-101.

Fisher, John. (1997): "The Challenge of Change: The Positive Agenda of the TGWU." International Journal of Human Resource Management, V. 8, n 6, pp. 797-806.

Godard, John. (1997): "Whither Strategic Choice: Do Managerial IR Ideologies Matter?",Industrial Relations, V. 36, nº 2, pp. 206-228.

Godard, John. 1997. "Whither Strategic Choice: Do Managerial IR Ideologies Matter?" Industrial Relations, V. 36, n $\mathrm{n}^{\circ}$ 2, pp. 206-228.

Guest, David. 1995. "Human Resource Management, Trade Unions, and Industrial Relations." en John Storey, (ed.), Human Resource Management: A Critical Text. London, Routledge.

Huselid, M. A. (1995): "The impact of human resource management practices on turnover, productivity, and corporate financial performance" Academy of $\mathrm{Ma}$ nagement Journal, n’38, pp 635-672.

Ichniowski, Casey, Shaw Kathryn, y Premushi Giovanna, (1997): "The Effects of Human Resources Management Practices on Productivity: A Study of Steel Finishing Lines.”,American Economic Review, V. 87, n. 3, pp. 291-313. 
Ichniowski, Casey, y Shaw Kathryn., (1998): The Effects of Human Resources Management Systems on Economic Performance: An International Comparison of U.S. and Japanese Plants.Mimeograph, Columbia University.

Juran J.M., (1989): Juran on Leadership for Quality, New York, NY Free press.

Kaoru Ishikawa (1980): QCCircle Koryo: General Principles of the QC Circle, QC Circle Headquarters (JUSE), Tokyo.

Kaoru Ishikawa (1985):How to Operate QC Circle Activities,Amer Society for Quality, Tokio.

Katz, Harry C., Kochan, Thomas A., y Gobeille Kenneth R., (1983): "Industrial Relations Performance, Economic Performance, and QWL Programs: An Interplant Analysis.”,Industrial and Labor Relations Review, Vol. 37, No. 1, pp. 3-17.

Katz, Harry C., Kochan, Thomas A., y Weber Mark A., (1985): “Assessing the Effects of Industrial Relations Systems and Efforts to Improve the Quality of Working Life on Organizational Effectiveness.", Academy of Management Journal, Vol. 28, No. 3, pp. 509-526.

Kizilos, Mark, y Reshef Yonatan., (1997): "The Effect of Workplace Unionization on Worker Responses to HRM Innovation.”,Journal of Labor Research, Vol. 18, No 4, pp. 641-656.

Koch, Marianne J., y McGrath Ruth G., (1996): "Improving Labor Productivity: Human Resource Management Policies Do Matter.",Strategic Management Journal, Vol. $17, \mathrm{~N}^{\circ} 5$, pp. 335-354.

Kochan, Thomas, (1995): "Using the Dunlop Report to Achieve Mutual Gains." Industrial Relations, Vol. 34, No 3, pp. 350-366.

Kochan, Thomas, y Osterman Paul., (1994): The Mutual Gains Enterprise. Boston, Harvard Business School Press.

Lawler, Edward (1992): The ultimate advantage: Creating the high-involvement organization, San Francisco, Jossey-Bass.

Lawler, Edward (1996): From the ground up: Six principles for building the new logic corporation, San Francisco, Jossey-Bass.

Levine, D., (1995) Reinventing the workplace: How business and employers can both win. Washington, DC: Brookings Institution.

Levine, David, y Tyson Laura. (1990): "Participation, Productivity, and the Firm's Environment." en Blinder, Alan S. (ed.), Paying for Productivity. Washington, D.C.: Brookings Institution Press.pp. 183-243. 
MacDuffie, John P., (1995): "Human Resource Bundles and Manufacturing Performance: Organizational Logic and Flexible Production Systems in the World Auto Industry.",Industrial and Labor Relations Review, Vol. 48, № 2, pp. 197-221.

Malcomson, James, (1983): "Trade Unions and Economic Efficiency", Economic Journal, $\mathrm{n}^{\circ} 93$, p 50-65.

Pfeffer, J. (1998): The human equation: Building profits by putting people first, Boston, Harvard Business School Press.

Roche, William K., y Geary John F., (2002): “Advocates, Critics, and Union Involvement in Workplace Partnership: Irish Airport.” British Journal of Industrial Relations, Vol. 40, No 4, pp. 659-688.

Rosen, Sherwin, (1993): "The Theory of Equalizing Di erences," in Handbook of Labor Economics, Volume 1, Amsterdam, Oxford and Tokyo: North-Holland, pp. 641-692.

Rubinstein, Saul, and Kochan Thomas., (2001): Learning from Saturn, Ithaca, N.Y: Cornell University Press.

Weitzman, Martin, y Douglas Kruse, (1990): "Profit Sharing and Productivity." en Alan (ed.), Paying for Productivity., Washington, D.C.: Brookings Institution, pp. $95-142$.

Wenchuan Liu, Guthrie, James P., Flood Patrick C., MacCurtain Sarah (2009): Unions and the adoption of high performance work systems: Does employment security play a role?,Industrial and Labor Relations Review, v.63, n¹, pp 109-127.

Wright, P. M., \& McMahan, G. C., (1992): Theoretical perspectives for strategic human resource management. Journal of Management, nº18, pp. 295-320. 